\title{
High subsonic flow of an organic vapor past a circular cylinder
}

\author{
Felix Reinker $^{1} \cdot$ Robert Wagner $^{1} \cdot$ Leander Hake $^{1} \cdot$ Stefan aus der Wiesche ${ }^{1}$
}

Received: 14 October 2020 / Revised: 30 December 2020 / Accepted: 1 February 2021

(c) The Author(s) 2021

\begin{abstract}
A circular cylinder was tested in the cross-flow of an organic vapor (Novec $\left.{ }^{\mathrm{TM}} 649\right)$ and of air over the subsonic $(M<0.4)$ and high subsonic $(0.4<M<0.8)$ speed range in a continuously running pressurized closed-loop wind tunnel test facility. Time-averaged pressure measurements gave information on surface pressure distributions, and the corresponding drag and base pressure drag coefficients were obtained. Due to the charging of the wind tunnel, different values of the compressibility factor $(0.876<Z<0.999)$ could be achieved for the organic vapor flow. This enabled in combination with the results for air an assessment of the impact of non-ideal gas dynamics on the form drag of a cylinder in the considered highly subsonic flow regime. The new experimental data were compared with available literature results. Changes in surface pressure distribution at higher subsonic velocities were identified and discussed. It was found that non-ideal gas effects did not strongly affect the overall drag. The variation of drag coefficient over the Mach number range was comparable with literature data for ideal-gas compressible flow, including shock-less and intermittent shock wave, and permanent shock wave flows regimes. At Mach 0.4 , the flow of Novec ${ }^{\mathrm{TM}} 649$ was in the shock-less regime and exhibited a pronounced dependency on the Reynolds number. An increase in drag was observed at Mach 0.6 which was attributed to the commencement of vortex shedding. Non-ideal thermodynamics only affected the flow locally and a reduction of the critical pressure coefficient in the high subsonic flow regime was observed in the surface pressure distribution. However, this mechanism did not alter significantly the overall drag behavior.
\end{abstract}

\section{Graphic abstract}

Drag coefficient $C_{D}$ against Re for several Mach numbers $M$ and comparison with available literature results obtained for air (colored symbols indicate different Mach number clusters)

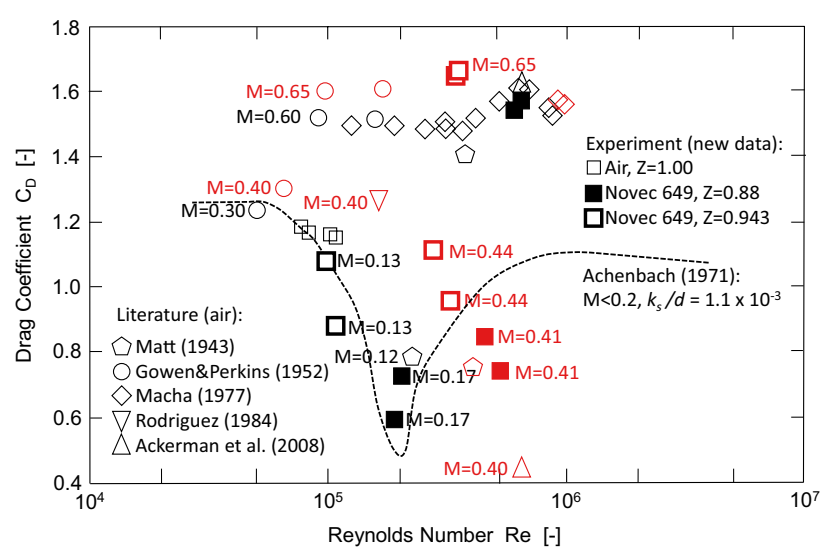

Stefan aus der Wiesche

wiesche@fh-muenster.de

1 Department of Mechanical Engineering, Muenster University of Applied Sciences, Stegerwaldstr, 3948565 Steinfurt, Germany

\section{Introduction}

The flow past a circular cylinder has been investigated intensively for more than a century, as demonstrated for instance by the two-volume monograph of Zdravkovich (2003). 
Numerous papers are now available in the open literature dealing with this fundamental flow configuration. This flow configuration is attractive for scientists because important fundamental flow phenomena like boundary layer transition and vortex shedding can be studied. Therefore, the circular cylinder is an obvious starting point for many technical applications including heat transfer from tubes, the flow past aircraft bodies, or even turbomachinery.

The majority of published papers have been concerned with the incompressible flow, and much less is known regarding high subsonic or supersonic flow past circular cylinders. Very early experimental studies were conducted by Matt (1943) and Gowen and Perkins (1952) using cylinders of different diameters placed in the test section of wind tunnels with air as working fluid. Later, Naumann and Pfeifer (1962), Macha (1977), Murphy and Rose (1978), and Rodriguez (1984) presented experimental data for a wide range of Mach and Reynolds numbers for the flow of air past a circular cylinder. Ackerman et al. (2008) investigated in detail the base pressure behavior of a circular cylinder in the high subsonic cross-flow of air as an idealized model for the study of trailing edge losses of turbine blades. In that context, MacMartin and Norbury (1974) concluded earlier "that the base pressure is an important quantity and calculation methods which neglect base pressure effects are incapable of accurately calculating the flow patterns or the total pressure loss." Despite the importance of trailing edge vortex shedding for turbine blading, the problem of predicting the base pressure is more widespread and generic, and the circular cylinder represents a canonical test object for studying such base pressure phenomena. The cylinder base pressure is known to be strongly dependent on the Mach number. At supersonic speeds, the low base pressure level is a result of shocks and expansions. This tends to be a relatively steady process. At subsonic speeds, shocks only play a role as the velocity reaches critical levels and, in general, the unsteady process of vortex shedding is more important.

Data on cylinder wall pressure distribution and drag in the high subsonic flow regime of non-ideal gas flows are essentially missing in literature so far. Although previous experimental studies have investigated the aerodynamics and the impact of non-ideal gas effects using thin airfoils placed in the test section of closed-loop test rigs working with real gas (see, for instance, Anders (1993)), the canonical configuration of a circular cylinder has not been considered yet in more detail. Non-ideal compressible flows of molecularly complex vapors are of interest for diverse industrial applications such as transportation of high-pressure fuels and chemicals and turbomachines operating with non-ideal fluids such as those found in ORC power systems and $\mathrm{SCO} 2$ power systems. It is hence not surprising that there is a recent trend to collect experimental data for non-ideal gas flows, as demonstrated by Cozzi et al. (2015) or Spinelli et al. (2018). In these studies, supersonic nozzle flows without blunt bodies in the flow domain were investigated.

To investigate flow phenomena related to immersed blunt bodies and base pressure phenomena under non-ideal conditions, an experimental study was conducted regarding pressure distribution and form drag of a circular cylinder placed in the test section of a closed wind tunnel facility using air and an organic vapor. This study concentrated on the subsonic up to the high subsonic flow regime (up to Mach 0.7), which is highly relevant for many technical applications. At supersonic Mach numbers, fundamental phenomena related to shock waves become dominant. This flow regime will be the subject of future research after clarifying high subsonic flow phenomena.

\section{Experimental method}

The experiments were conducted in a continuously running closed-circuit wind tunnel capable of operating with air and an organic vapor at elevated pressure and temperature levels as described in more detail in the following section. The use of this test facility enabled-with certain limitations-the detailed investigation of potential non-ideal gas effects.

\subsection{Working fluid selection}

In the case of compressible flow experiments, the following relevant similarity numbers are typically quoted (see, for instance, Dejc and Trojanovskij (1973)): the Mach number $M=u / a$, the Reynolds number $\operatorname{Re}=\rho \bullet u \bullet L / \mu$, the isentropic exponent $\kappa=c_{p} / c_{v}$, and the Prandtl number $\operatorname{Pr}=c_{p} \bullet \mu / \lambda$, which is of special interest for heat transfer phenomena. It should also be noted that in the case of expanding non-ideal gas flows the isentropic exponent $\kappa$ is not a simple constant. In addition to the ideal gas expression $\kappa=c_{p} / c_{v}$, it is possible to distinguish between three different definitions depending on the considered isentropic relations (see Kouremenos (1986)). However, in the present study, a further quantity has to be introduced to quantify non-ideal gas behavior. Here, the compressibility factor $Z$ was selected as an additional non-dimensional number, and the working fluids were thus characterized by two dimensionless quantities $\kappa$ and $Z$.

Experiments with dry air with a compressibility factor $Z$ $\approx 1.00$, representing a compressible ideal gas flow, served as the datum for the new measurements of a non-ideal gas flow past a circular cylinder. For dry air, valuable data for pressure distribution and drag are available in the literature, but new measurements were conducted to validate the applied experimental measurement technique, too. As discussed by Reinker et al. (2019), the perfluorinated ketone Novec ${ }^{\mathrm{TM}} 649$ by $3 \mathrm{M}^{\mathrm{TM}}$ is a suitable fluid for investigating non-ideal gas 
effects in laboratory test facilities, and it was already used for the design of a waste heat recovery ORC system (see Cogswell et al. (2011)). In the present study, flow measurements at two different average density levels of Novec ${ }^{\mathrm{TM}} 649$ were conducted. The average density level is defined as the ratio of the inventory mass of working fluid to the total flow domain volume. Due to this kind of charging, a low-density case, characterized by a compressibility factor $Z=0.945$ (close to unity), and a high-density case, characterized by a compressibility factor $Z=0.876$ (significantly lower than unity), were reached. The former case represented an ideal gas flow regime of the organic vapor, whereas the latter case corresponded to a non-ideal gas flow regime. For both lowand high-density case, the values for the isentropic exponent $\kappa$ of Novec ${ }^{\mathrm{TM}} 649$ were nearly identical but much lower than for air $\left(\kappa \approx 1.05\right.$ for Novec ${ }^{\mathrm{TM}} 649$ in comparison to $\kappa=1.40$ for dry air, respectively). This enabled an examination of the impact of the compressibility factor $Z$ on the flow past a circular cylinder. Table 1 lists typical values for thermophysical properties of the working fluids at representative pressure and temperature levels obtained during the experiments. In this study, all thermophysical material properties were calculated utilizing the REFPROP database (NIST, standard version 9.0, see also McLinden et al. (2015) for more details regarding the thermodynamics, equations of states, and uncertainty levels) using pressure and temperature values, as measured in the experiments.

\subsection{Wind tunnel test facility and procedure}

The present experiments were performed in the high subsonic flow test section of the continuously running closedloop organic vapor wind tunnel (CLOWT) at Muenster University of Applied Sciences, see Fig. 1a. Design features of the test facility CLOWT and its working principle were discussed in previous publications (Reinker et al. (2018, 2019), , ). After passing the compressor with adjustable running speed $n$, the working fluid was decelerated in the diffuser and entered the settling chamber equipped with a chiller where total pressure $p_{0}$ and total temperature $T_{0}$ were measured. To ensure steady-state operation, a chiller was used to remove the dissipated compressor power. The actual operation temperature level $T_{0}$ was held constant by controlling the coolant water flow of the chiller as part of the control system. During start-up, the entire test facility was electrically heated up to the desired operating temperature. The working fluid mass flow rate $\dot{m}$ was recorded through a mass flow device in the subsonic return of the closed wind tunnel where also pressure and temperature values were recorded for control purposes.

The test facility CLOWT was designed as a pressure vessel with a two-stage contraction. The first subsonic piece-wise conical axisymmetric nozzle (from nominal diameter $500 \mathrm{~mm}$ to $250 \mathrm{~mm}$ ) was part of the outer shell structure, and was characterized by a moderate contraction ratio of about 3.7 , see Fig. 1a. Details about this nozzle and its performance were published recently by Hasselmann et al. (2019). This incompressible flow nozzle is permanently installed in CLOWT, and the desired higher Mach numbers can be achieved by individually designed secondary nozzles installed in the long basic test section pipe. During the present experiments, a second subsonic nozzle and a (specific) test section equipped with a circular cylinder as a test object were placed in this basic test section, see Fig. 1b. The second contraction was achieved by a three-dimensional nozzle based on additive manufacturing (SLM) providing the cross-sectional change from round to rectangular contraction (from nominal diameter $250 \mathrm{~mm}$ to a rectangular cross-section $50 \mathrm{~mm} \times 100 \mathrm{~mm}$ ) leading to a total contraction ratio of about 38 . The second nozzle was designed to provide high subsonic flows up to $M=0.7$ in the test section. Details about the nozzle design based on a Lamé super-ellipse can be found elsewhere (Passmann et al. (2017)). In the high-speed test section, the free static pressure $p$ was measured through end wall pressure taps. The surface wall pressure $p_{w}$ of the circular cylinder was measured by a small pressure hole, see Fig. 1b.
Table 1 Thermophysical properties of dry air and Novec $^{\mathrm{TM}} 649$ at representative pressure and temperature levels (calculated using REFPROP 9.0)

\begin{tabular}{lccc}
\hline Fluid & Air & $\begin{array}{c}\text { Novec } \\
\text { Experiment } 1\end{array}$ & $\begin{array}{c}\text { Novec } \\
\text { Experiment } 249\end{array}$ \\
\hline Pressure level $p[\mathrm{MPa}]$ & 0.511 & 0.147 & 0.310 \\
Temperature level $T[\mathrm{~K}]$ & 307.5 & 370.0 & 370.0 \\
Density $\rho\left[\mathrm{kg} / \mathrm{m}^{3}\right]$ & 5.796 & 15.983 & 36.354 \\
Isentropic exponent $\kappa=\mathrm{c}_{\mathrm{p}} / \mathrm{c}_{\mathrm{v}}[-]$ & 1.408 & 1.039 & 1.056 \\
Compressibility factor $Z[-]$ & 0.999 & 0.945 & 0.876 \\
Fundamental derivative $\Gamma[-]$ & 1.204 & 0.980 & 0.975 \\
Speed of sound $a[\mathrm{~m} / \mathrm{s}]$ & 352.13 & 94.89 & 88.08 \\
Dynamic viscosity $\mu[\mathrm{Pa} \mathrm{s}]$ & $1.896 \times 10^{-5}$ & $1.391 \times 10^{-5}$ & $1.388 \times 10^{-5}$ \\
Prandtl number $\operatorname{Pr}[-]$ & 0.7092 & 0.7587 & 0.7680
\end{tabular}


Fig. 1 Closed-loop organic vapor wind tunnel test facility (CLOWT): a overview and basic instrumentation. $\mathbf{b}$ details of the test section

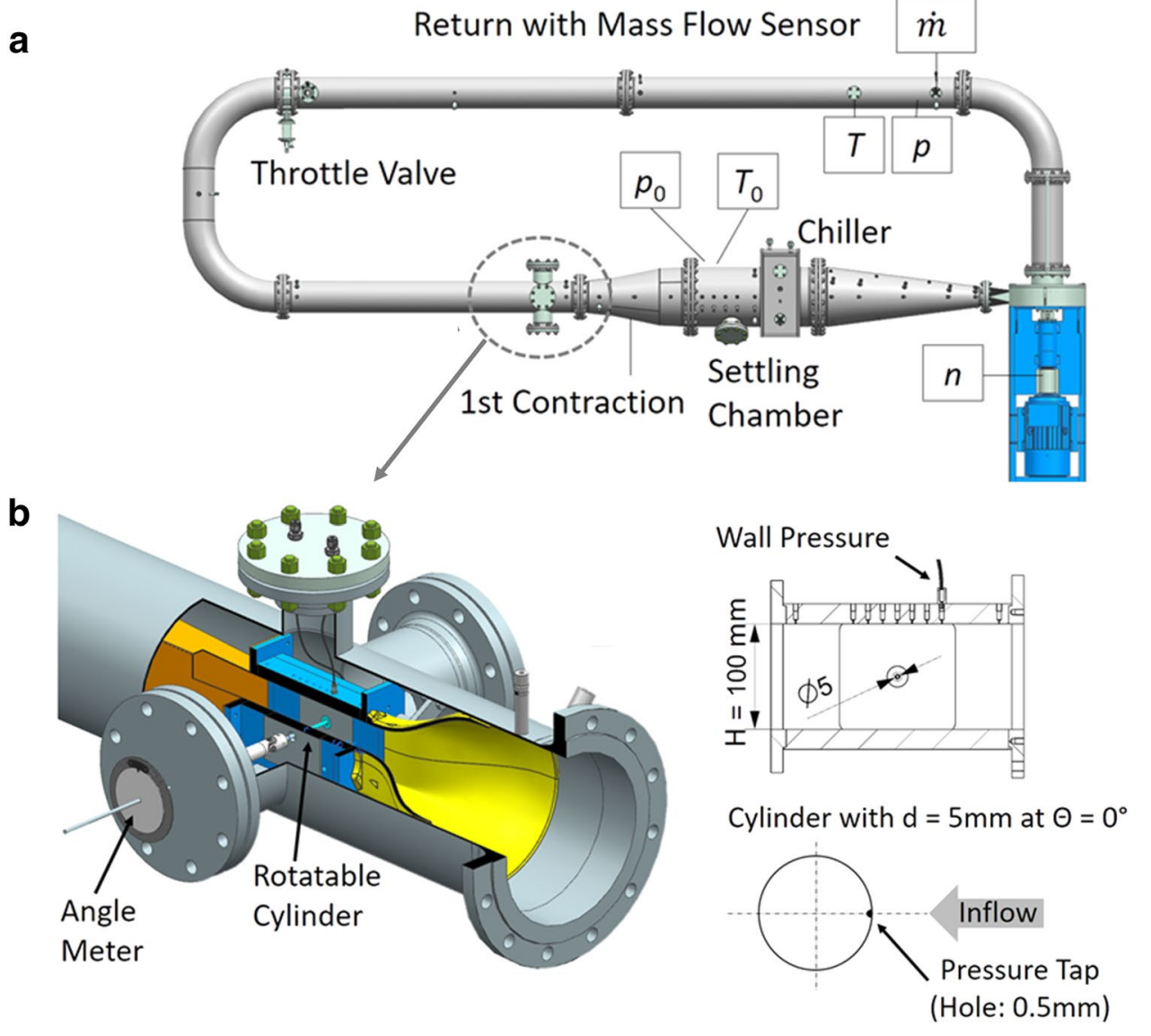

The circular cylinder with diameter $d=5 \mathrm{~mm}$ and span $50 \mathrm{~mm}$ was mounted in the center of the test section, see Fig. 1 b. At first sight, this cylinder diameter seems to be rather small, but due to the high vapor density $\rho$ of Novec ${ }^{\mathrm{TM}} 649$, relatively high Reynolds numbers $\operatorname{Re}=1 \cdot 10^{5}-6.1 \cdot 10^{5}$ resulted even for this small test object during the present experiments. In addition to subcritical flow, critical and supercritical Reynolds numbers were achieved during the present experiments. The area blockage ratio was $\mathrm{d} / H=0.05$ with $H=100 \mathrm{~mm}$ as test section height. The small size of the cylinder in the test section avoided serious wall interference effects in the high-subsonic flow regime. There was no need to employ slotted walls during the present experiments because the Mach number level was well below unity. The cylinder, as shown in detail in Fig. 2, was equipped with a small pressure hole of $0.5 \mathrm{~mm}$ in diameter for measuring the local cylinder wall pressure $p_{w}$. This wall pressure would be equal to the total pressure $p_{0}$ at the cylinder stagnation point (at a circumferential angle $\theta=0^{\circ}$ ) in the case of isentropic flow. The good agreement of the settling chamber total pressure and the cylinder stagnation point wall pressure confirmed the assumption of an isentropic nozzle flow in the contraction zone. The cylinder could be fully rotated about its circumferential angle $\theta$ (i.e., $0^{\circ} \leq \theta \leq 360^{\circ}$ ). This enabled a determination of the entire circumferential pressure distribution $p_{w}(\theta)$, but due to the finite size of the pressure hole, the actual value of $p_{w}(\theta)$ has to be interpreted as an area-averaged value covering a circumferential sector size of order $\Delta \theta=5.7^{\circ}$. That represents also the resolution in terms of circumferential angles.

\subsection{Inflow turbulence and cylinder roughness}

From incompressible flow, it is well known, see Achenbach (1971), that cylinder roughness and inflow turbulence level can affect pressure distribution and drag significantly. Initially, it was planned to employ a hydraulically smooth cylinder, but measurements (see also the corresponding discussion in Sect. 3) indicated that the actual stainless-steel cylinder surface was better characterized by a relative roughness of order $k_{s} / d \approx 1 \times 10^{-3}$ with $k_{s}$ as equivalent sand grain roughness size even after a careful polishing process. Due to the small cylinder diameter $d$, that "rough" level resulted although the absolute roughness parameter values of the cylinder surface were low. A photograph of the cylinder surface is shown in Fig. 2a. The roughness structure was aligned with the main flow direction, but for the pressure hole, two imperfections could not be avoided which are shown in 

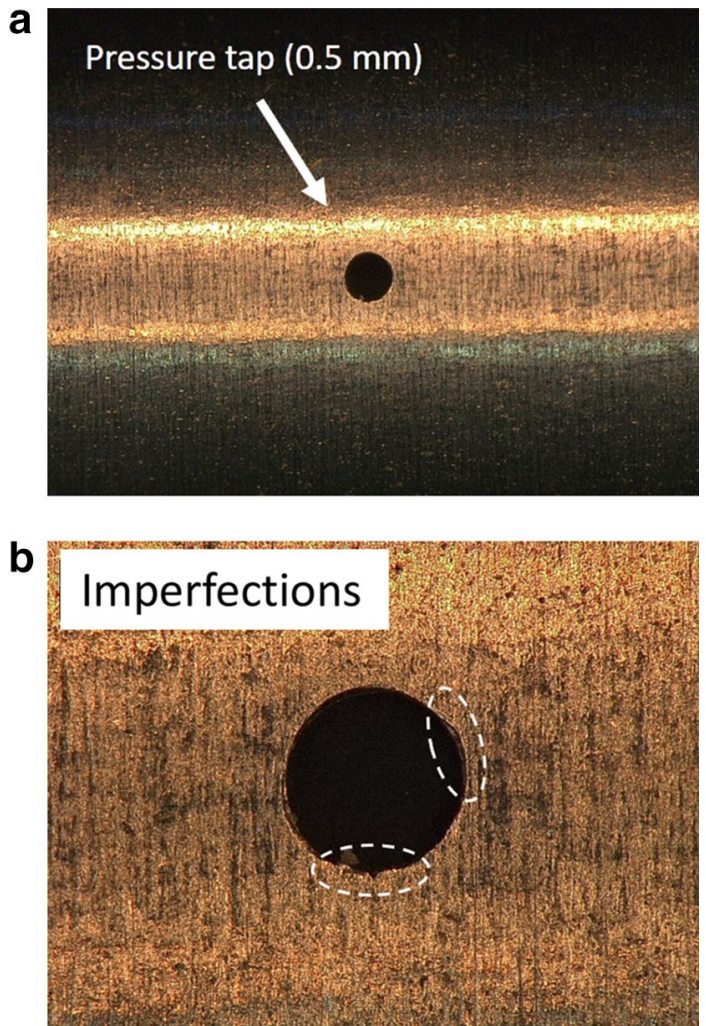

Fig. 2 Photographs of the actual cylinder test object: a surface structure and $\mathbf{b}$ detailed view of the imperfections of the pressure hole

Fig. 2b. These small imperfections were insignificant for the majority of the measurements; the exception being at flow transition points, where an effect was observed due to the flow being highly sensitive to any disturbance. It was found that a weak asymmetry of the surface pressure distribution $p_{w}(\theta)$ could occur at such a transition point for measurements performed for the lower circumferential interval $0<\theta<180^{\circ}$ as well as for the upper interval $360^{\circ}>\theta>180^{\circ}$. In the case of a perfect cylinder without any imperfections, the pressure distributions would be identical for both circumferential intervals, but at a transition point, it was observed that measurable deviations could occur. These deviations were explained as a kind of "trip-wire effect" caused mainly by the bottom imperfection shown in Fig. 2 b.

The actual inflow turbulence level $T u$ was measured utilizing hot-wire anemometry in the test section. In a prior study, a single hot wire probe (manufactured by SVM $\mathrm{GmbH}$, Stuttgart) with wire diameter $10 \mu \mathrm{m}$ and wire length $4 \mathrm{~mm}$ was placed instead of the cylinder, and turbulence quantities were obtained for both working fluids, see Reinker and aus der Wiesche (2020) for more details about hot-wire measurements in organic vapors. It was found that the inflow turbulence level of the present cylinder measurement campaigns with its limitations to high subsonic Mach numbers was of order 0.5 up to $0.7 \%$, see Fig. 3. This level was well

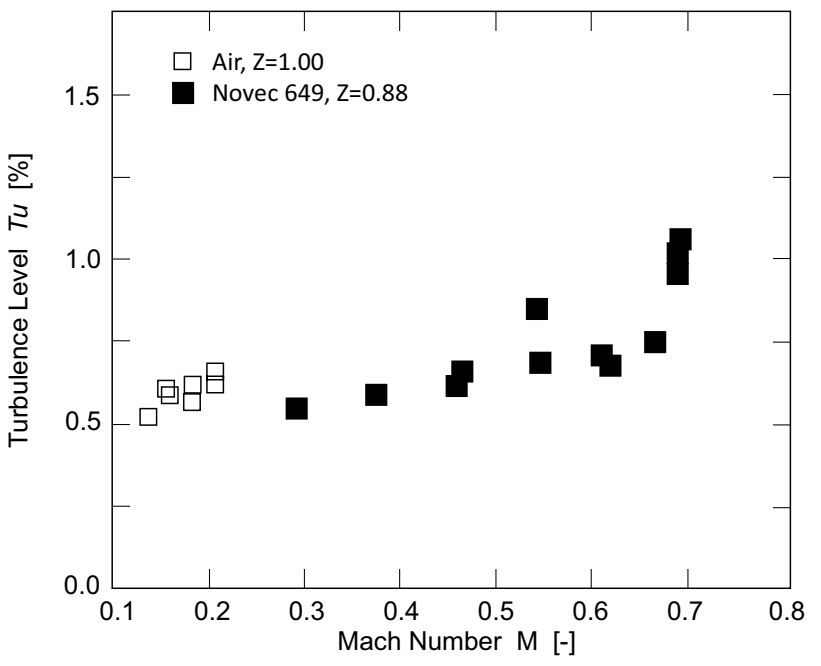

Fig. 3 Inflow turbulence level $T u$ for air and Novec ${ }^{\text {TM }} 649$ within the test section against Mach number $M$

comparable with the conditions reported by Achenbach (1971) for his study of incompressible flow past rough cylinders (he reported a value of $0.7 \%$ ). A systematic effect of the Mach number $M$ on the turbulence level Tu was observed, as indicated by the tendency of the data points plotted in Fig. 3. The turbulence level tended to increase as the Mach number increased.

The turbulent micro length $\lambda$ in the high-speed test section was determined through hot-wire measurements and obtaining the autocorrelation $f(r / \lambda)$ for the normalized distance $r / \lambda$. The distance $r$ was calculated using the mean velocity $u_{m}$ and the time delay $\Delta \tau$ assuming the Taylor hypothesis. It was found that the shape of the autocorrelation $f(r / \lambda)$, Fig. 4a, was for both fluids in good agreement with theoretical predictions for isotropic turbulence, see Rotta (1972). The assumption of isotropic turbulence in the test section was also supported by the decrease in the power spectrum density by a power law with exponent $-5 / 3$. The calculated normalized turbulent length scale $\lambda / D_{h}$ with $D_{h}$ as the hydraulic diameter of the test section with a rectangular cross-section $(100 \mathrm{~mm} \times 50 \mathrm{~mm})$ is plotted against the Mach number in Fig. 4b. The observed level of $\lambda / D_{h}$ was in good agreement with literature results for pipe flows, Schlichting and Gersten (1997). However, this means that the turbulent micro length $\lambda$ was of order $5 \mathrm{~mm}$ up to $10 \mathrm{~mm}$ which was at the same level (or even higher) than the cylinder diameter $d$.

\subsection{Data reduction and uncertainty analysis}

Each operation point was defined by a Reynolds number $\operatorname{Re}=u \cdot d \cdot \rho / \mu$, a Mach number $M=u / a$, an isentropic exponent $\kappa$, and a compressibility factor $Z$. The values for $Z$ and $\kappa$ and other thermodynamic properties like the speed of sound $a$ 

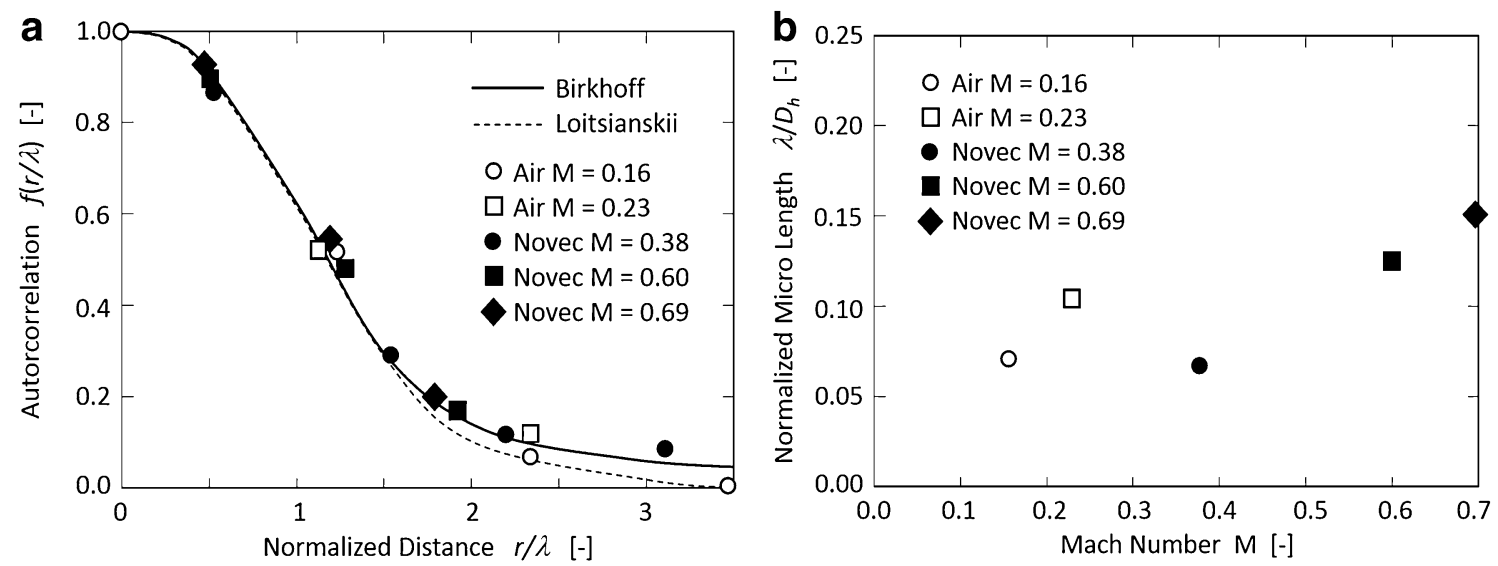

Fig. 4 Autocorrelation $f$ as a function of normalized radial distance $r / \lambda$ (a) and normalized micro length scale $\lambda / D_{h}$ against Mach number $M$ (b) in the test section

or density $\rho$ were calculated employing REFPROP using the measured static pressure $p$ in the test section and the calculated static temperature $T$, see McLinden et al. (2015). For data reduction, a one-dimensional isentropic analysis was completed based on the measured total conditions $p_{0}$ and $T_{0}$ in the settling chamber, the measured mass-flow rate $\dot{m}$, and the static pressure $p$ inside the test section to derive temperature and velocity. Since the static pressure $p$, mass flow rate $\dot{m}$ and the (total) pressure $p_{0}$ and temperature $T_{0}$ in the settling chamber delivered higher accuracy, this method was preferred instead of using the (total) temperature in the high-speed test section. The latter one was stronger disturbed by thermal noise from the environment and there was also uncertainty regarding the appropriate recovery factor for organic vapors. The assumption of an essentially isentropic nozzle flow through the contraction zone was checked by a comparison of the settling chamber pressure $p_{0}$ with the cylinder pressure at the stagnation point position, i.e., $p_{w}\left(\theta=0^{\circ}\right)$, see Fig. 1b. The deviations between $p_{0}$ and $p_{w}\left(\theta=0^{\circ}\right)$ remained within the experimental uncertainty of the employed pressure transducers.

The time-averaged pressure coefficient

$C_{p}(\theta)=\frac{p_{w}(\theta)-p}{p_{0}-p}$

was calculated using the time-averaged cylinder wall pressure $p_{w}$, the static pressure $p$ in the test section, and the total pressure $p_{0}$ in the settling chamber. After setting the respective circumferential angle in the experiment, it took about $5 \mathrm{~s}$ up to $10 \mathrm{~s}$ to achieve steady-state conditions inside of the pressure lines. After achieving steady-state conditions, the pressure was measured. The measurements were taken with a sampling rate of two seconds and a minimum measurement time of $10 \mathrm{~s}$ to record statistically independent samples.
The profile drag coefficient $C_{D}$ was calculated using the time-averaged pressure coefficient values $C_{p}(\theta)$ obtained for one half of the cylinder. Since the cylinder was rotated about the entire circumferential range, values for the two halfintervals were obtained for each operation point (namely for $0 \leq \theta \leq 180^{\circ}$ and $180 \leq \theta \leq 360^{\circ}$, respectively). For instance, the drag coefficient $C_{D}$ for the upper half was calculated through numerical integration of the expression

$C_{D}=\int_{180^{\circ}}^{0^{\circ}} C_{p}(\theta) d \sin \theta$

In addition to the above drag coefficient $C_{D}$, a base pressure drag coefficient $C_{B D}$ was calculated through

$C_{D B}=\int_{180^{\circ}}^{\theta_{\text {sep }}} C_{p}(\theta) d \sin \theta$

as done by Ackerman et al. (2008) using the circumferential position of flow separation $\theta_{\text {sep }}$. The circumferential position of flow separation was determined employing a criterion proposed by Stratford (1959) for two-dimensional turbulent boundary layers. In the case of laminar boundary layers, flow separation starts shortly after the minimum surface pressure location. In the case of turbulent boundary layers, the separation point is within the region of increasing surface pressure, and Stratford's criterion enables an estimation of the distance between minimum surface pressure location and flow separation. Equation (2) includes contributions of form drag and neglects skin friction and drag created by the loss of total pressure across local shock waves emanating from the cylinder surface. In the case of incompressible flow, the contribution to profile drag from skin friction drag is very small. In high subsonic flow the contribution to drag by 
total pressure loss through shocks remains small (although it increases).

Experiments with organic vapor flow at elevated pressure and temperature levels require special efforts, and a discussion of the measurement uncertainty level is of major importance since no standard instrumentation and well-established procedures can be applied so far.

The uncertainty of the flow variables can have two uncertainty sources in general, namely bias $B_{x}$ and precision $P_{x}$. The total uncertainty of a variable $X$ is a combination of both

$\frac{\Delta X}{X}=\sqrt{\left(\frac{B_{x}}{X}\right)^{2}+\left(\frac{P_{x}}{X}\right)^{2}}$

The quantification of the uncertainty due to bias errors of a variable $X$ depending on quantities $x_{1}, x_{2}$, was determined by using

$\frac{B_{x}}{X}=\sqrt{\left(\frac{\partial X}{\partial x_{1}} \frac{B_{x 1}}{X}\right)^{2}+\left(\frac{\partial X}{\partial x_{2}} \frac{B_{x 2}}{X}\right)^{2}+\ldots}$

where the $B_{x i}$ is the bias error or the uncertainty of a given measurement device $i$. Typically, that uncertainty is provided in some percent of the full scale of the device. Similarly, the general expression for the precision uncertainties calls

$\frac{P_{x}}{X}=\sqrt{\left(\frac{\partial X}{\partial x_{1}} \frac{P_{x 1}}{X}\right)^{2}+\left(\frac{\partial X}{\partial x_{2}} \frac{P_{x 2}}{X}\right)^{2}+\ldots}$

where the precision errors $P_{x i}$ were given by means of

$P_{x i}=\frac{\tau}{\sqrt{N}} S_{i}$

where $N$ is the number of samples (values of $N=5$ up to 20 were chosen for the pressure and temperature measurements for each operation point during the present study), $\tau$ is the so-called Student-t-factor for the given confidence interval (here, a value of about $\tau=2.1$ for $95 \%$ confidence level was assumed), and $S_{i}$ is the standard deviation of the sample values $x_{i 1}, x_{i 2}, \ldots, x_{i N}$.

Although the uncertainty level of the employed pressure transducers was of order 0.1 up to $0.2 \%$ (depending on the actual pressure level), a much higher total uncertainty level (of order $\Delta p / p=0.5$ up to $1.6 \%$ ) resulted. The reason for the higher uncertainty level was given by the bias error caused by condensation in the pressure lines. That problem and its technical consequences yielded an additional measurement error which is described in more detail by Reinker et al. (2020). In the present case, the condensation issue was the main source of uncertainty for the pressure measurements, and the contribution due to precision was nearly negligible (the precision error was of order 0.01 up to $0.05 \%$ ).
The absolute uncertainty of the temperature measurements (using temperature sensors PT100 1/10 DIN B) was of order $\Delta T=0.1 \mathrm{~K}$ (nearly independent of the actual temperature level) resulting in a total relative uncertainty level of $\Delta T / T=0.06$ up to $0.1 \%$ including the data logger and precision contributions. The contribution due to precision was only of order $0.01 \%$ regarding temperature measurements.

In addition to the general uncertainty sources for the primary variables pressure and temperature as outlined above, the calculation uncertainty of the thermodynamic properties had to be considered due to the selected equation of states and fluid database (i.e., REFPROP) and the stability of the test facility operating point during a measurement run. The calculation of thermodynamic variables based on the REFPROP database with a suitable equation of states. REFPROP provided information about the (estimated) uncertainty range of thermodynamic variables, and these uncertainties were treated as bias errors in the uncertainty analysis. For example, REFPROP quoted that the uncertainty in vapor speed of sound $a$ for Novec ${ }^{\mathrm{TM}} 649$ was only $0.05 \%$, and this value was used as corresponding bias error contribution in the calculation of the uncertainty of the Mach number. A substantial source of uncertainty was given by the uncertainty regarding the density calculation which affected also the calculation of the Reynolds number. Without considering the systematic error due to the finite accuracy of the thermodynamic equation of states, the total relative uncertainty level for the density was of order $\Delta \rho / \rho=0.7$ up to $2.0 \%$ (depending on the pressure and temperature level). Taking into account the systematic uncertainty due to the thermodynamic equation of states as quoted by REFPROP, a higher uncertainty level resulted of about $\Delta \rho / \rho=1.6$ up to $3.0 \%$. The uncertainty level of the density affected the total uncertainty of the mass flow rate, and hence a relative uncertainty of order 1.6 up to $4.5 \%$ resulted for that quantity. That uncertainty directly affected the Mach and the Reynolds number uncertainty levels for which similar figures were obtained. The uncertainty level of the compressibility factor $Z$ was obtained by inserting the minimum and maximum values into the thermodynamic equation of states.

In Table 2, the resulting uncertainty levels are listed at three representative operation points. The actual uncertainty in $C_{p}$ was higher in the case of air than in the case of Novec $^{\mathrm{TM}} 649$ because the employed pressure transducers had a better uncertainty performance for higher dynamic loads. The circumferential resolution $\Delta \theta$ was only moderate (of order $5.7^{\circ}$, see Sect. 2.2) due to the small cylinder diameter $d=5 \mathrm{~mm}$ and the finite pressure hole diameter $0.5 \mathrm{~mm}$.

During operation, the nominal operation point pressure and temperature values scattered slightly over time due to the non-perfect temperature control loop with its delaytime behavior. Although the measurement uncertainty of the temperature sensors was of order $0.1 \mathrm{~K}$, the operation 
Table 2 Total measurement uncertainty levels for typical operation points

\begin{tabular}{|c|c|c|c|}
\hline Fluid & Air & $\begin{array}{l}\text { Novec }{ }^{\mathrm{TM}} 649 \\
\text { Experiment } 1\end{array}$ & $\begin{array}{l}\text { Novec }^{\mathrm{TM}} 649 \\
\text { Experiment } 2\end{array}$ \\
\hline Nominal pressure $p[\mathrm{MPa}]$ & 0.511 & 0.147 & 0.310 \\
\hline $\begin{array}{l}\text { Nominal temperature } T \\
{[\mathrm{~K}]}\end{array}$ & 307.5 & 370.0 & 370.0 \\
\hline Pressure $\Delta p / p$ & $0.4 \%$ & $1.4 \%$ & $0.6 \%$ \\
\hline Temperature $\Delta T / T$ & $0.06 \%$ & $0.10 \%$ & $0.10 \%$ \\
\hline Mach number $\Delta M / M$ & $2.6-4.9 \%$ & $1.0-4.0 \%$ & $1.0-3.0 \%$ \\
\hline Reynolds number $\Delta \operatorname{Re} / \operatorname{Re}$ & $2.0-4.0 \%$ & $1.8-4.9 \%$ & $1.8-4.8 \%$ \\
\hline $\begin{array}{l}\text { Compressibility factor } \\
\Delta Z / Z\end{array}$ & $<0.1 \%$ & $2.0 \%$ & $2.4 \%$ \\
\hline $\begin{array}{l}\text { Pressure Coefficient } \Delta C_{p} / \\
C_{p}\end{array}$ & $3.1 \%$ & $1.8 \%$ & $1.0 \%$ \\
\hline
\end{tabular}

Table 3 Maximum systematic operation point fluctuations during single measurement runs

\begin{tabular}{llll}
\hline Fluid & Air & $\begin{array}{l}\text { Novec } \\
\text { Experiment } 1\end{array}$ & $\begin{array}{l}\text { Novec } \\
\text { Experiment 2 }\end{array}$ \\
\hline Nominal pressure $p[\mathrm{MPa}]$ & 0.511 & 0.147 & 0.310 \\
Nominal temperature $T[\mathrm{~K}]$ & 307.5 & 370.0 & 370.0 \\
Mach number $\Delta M / M$ & $0.6 \%$ & $0.6 \%$ & $0.6-2.0 \%$ \\
Reynolds number $\Delta \mathrm{Re} / \mathrm{Re}$ & $0.5 \%$ & $0.8 \%$ & $0.8-2.5 \%$ \\
Compressibility factor $\Delta Z / Z$ & $<0.1 \%$ & $0.3 \%$ & $1.1 \%$ \\
Pressure Coefficient $\Delta C_{p} / C_{p}$ & $2 \%$ & $1 \%$ & $1 \%$ \\
\hline
\end{tabular}

temperature level could only be kept constant within $\pm 1 \mathrm{~K}$ during long-time operation over a day. Since a complete measurement run of the circumferential pressure distribution required some finite time, additional systematic fluctuations of the Mach and Reynolds numbers and the compressibility factor occurred for each single measurement run. In Table 3, the maximum values of these systematic fluctuations are listed. They were lower than the experimental uncertainty levels listed in Table 2, but in the case of a transition regime, when the flow was rather sensitive to disturbances, these fluctuations might be of some relevance.

\subsection{Blockage corrections}

Following Wyler (1975), the blockage effect can be looked upon as a perturbation of the velocity in the vicinity of the cylinder. The blockage parameter $\varepsilon=\delta u / u$ is defined as the ratio of the perturbation velocity $\delta u$ to the undisturbed free stream velocity $u$. The perturbation velocity $\delta u$ is the difference between the obtained velocity $u$ and the corrected value $u_{\text {cor }}$ which would be the true value in an ideal experiment with vanishing area blockage ratio $d / H$. There is a large body of literature available about blockage corrections, and a detailed review is beyond the scope of the present paper. However, it is useful to discuss the potential impact of blockage corrections on the presentation of results to assess the uncertainty related to wall interference effects.

To correct for wall interference effects, Roshko (1961) made use of the formulas of Allen and Vincenti (1944), which give, for the corrected values of velocity and drag coefficient $u_{\text {cor }}$ and $C_{D \text { cor }}$ in terms of the measured values $u$ and $C_{D}$,

$\frac{u_{\mathrm{cor}}}{u}=1+\frac{1}{4} C_{D}\left(\frac{d}{H}\right)+0.82\left(\frac{d}{H}\right)^{2}$
$\frac{C_{D \text { cor }}}{C_{D}}=1-\frac{1}{2} C_{D}\left(\frac{d}{H}\right)-2.5\left(\frac{d}{H}\right)^{2}$

and for the pressure coefficient $C_{p}$

$C_{p \mathrm{cor}}-1=\left(\frac{u}{u_{\mathrm{cor}}}\right)\left(C_{p}-1\right)$

These formulas were obtained by using image doublets to represent the interference between wall and cylinder, and image sources to represent the interference between wall and wake. Such an analysis does not take into account possible interference effects on the separation mechanism and the structure of the wake close behind the cylinder.

Wyler (1975) proposed based on new experimental data for cylinder probes placed in a stream of air at various Mach numbers

$\frac{\left|u-u_{\mathrm{cor}}\right|}{u}=\frac{C_{D}}{2\left(1-\mathrm{M}^{2}\right)}\left(\frac{d}{H}\right)$

Equation (10) represents an extension of the classical Maskell expression for blunt bodies concerning compressibility effects. In its incompressible form $(M=0)$, the blockage effect predicted by Eq. (10) is twice the magnitude of that predicted by purely analytical solutions (8). In the case of higher (subsonic) Mach numbers, the correction (10) predicts even higher values, and would even diverge at $M=1$.

An application of the above corrections demonstrates that blockage effects might be of some interest in comparisons with literature data although the actual area blockage ratio $d / H=0.05$ was low in the present study. For example, assuming representative values $C_{D}=1.1, M=0.2$, and $C_{p}=-1.0$, the two methods (as used by Roshko and Wyler) predicted corrected pressure coefficient values of $C_{\text {pcor }}=-0.94$ and -0.89 , respectively. The drag correction effect was of order 3 up to $5 \%$ for both methods. Hence, the impact of these corrections was comparable with the experimental uncertainty level. Since no definitive answer can be given to the question about the correct blockage corrections for non-ideal compressible flow, the above corrections might be interpreted as an additional bias effect. 


\subsection{Limitations and weaknesses}

The present experimental approach and procedure caused some limitations and weaknesses which should be briefly considered to provide a fair assessment of this study.

Ideally, the relevant similarity numbers $M$ and Re would be independently varied through their entire range for all selected values of $\kappa$ and $Z$. The value of the isentropic exponent $\kappa$ was well defined through the working fluid selection (Novec ${ }^{\mathrm{TM}} 649$ and air), but in the actual experiments, the Reynolds and the Mach numbers and the compressibility factor could not be modified independently. The compressor running speed affected directly both Reynolds number $\operatorname{Re}=u \cdot d \cdot \rho / \mu$ and Mach number $M=u / a$ through the mean velocity level $u$ which was governed by the compressor volume flow rate. The actual value of the Reynolds number Re could be changed by the average density $\rho$ (i.e., by charging of the wind tunnel), but this approach affected the pressure level and hence the compressibility factor in the case of Novec ${ }^{\mathrm{TM}} 649$ as well. The alternative option, to vary the Reynolds number Re utilizing different cylinder diameters $d$ was not feasible due to the expected wall interference for larger cylinders in the test section; the chosen value of $d=5 \mathrm{~mm}$ was also at the lower limit because otherwise, the relative roughness would be too high for a smaller cylinder. Consequently, it was not possible to investigate the entire field of similarity numbers separately, and a correlation between the Reynolds and the Mach numbers existed as in the case of previous studies (e.g., Gowens and Perkins (1952) or Rodriguez (1984)).

Due to serious financial constraints, it was not possible to buy and employ fast pressure transducers to resolve temporal pressure fluctuations, as done by Rodriguez (1984), and only time-averaged pressure values were recorded. An additional hot wire probe was not used together with the cylinder in the test section because the disturbance of the flow was found to be substantial: The stem diameter of the hot-wire probe was of the same order as the test cylinder itself, which means that a special kind of self-influencing tandem configuration would result for a setup with both cylinder and hot-wire probe in the test section.

\section{Results and discussion}

In the first set of experiments, low-speed results (i.e., $M<0.2$ ) were gathered to assess the accuracy of the present experimental approach. Then, the Mach number $M$ was increased, and essentially new data were obtained for the high-subsonic flow of an organic vapor past a circular cylinder.

\subsection{Low-speed results}

In the case of low-speed flow past a circular cylinder, the relevant similarity number is the Reynolds number, but it is also well known that surface roughness and inflow turbulence affect the flow. Since essentially incompressible flow can be assumed for $M<0.2$, the working fluid selection (i.e., air or Novec ${ }^{\mathrm{TM}}$ 649) should not be relevant for cylinder pressure distributions or drag coefficients.

In Fig. 5, some typical low-speed results for the pressure coefficient $C_{p}$ against circumferential angle $\theta$ are shown. Although the qualitative behavior of $C_{p}(\theta)$ was following the expectations, some deviations to literature data obtained at higher Reynolds numbers and using a smooth cylinder could still be observed in Fig. 5. The new data plotted in Fig. 5 were obtained at a much lower Reynolds number level, but they seemed to be comparable to the literature results for a smooth cylinder obtained at a much higher Reynolds number. It was argued that this observation was an effect of cylinder surface roughness. That could be confirmed by comparison with literature data obtained for a cylinder with similar relative roughness, see Fig. 6 . The agreement between the actual air measurements and the literature data published by Achenbach (1971) for a rough cylinder was quite reasonable. The deviations with the Novec ${ }^{\mathrm{TM}} 649$ data plotted in Fig. 6 might be explained by pronounced Reynolds number or surface roughness effects at the transition region, as discussed in the following.

Using the cylinder surface pressure distributions, the form drag coefficient $C_{D}$ was obtained for air and Novec ${ }^{\mathrm{TM}} 649$, and the low-speed results are shown in Fig. 7 together with

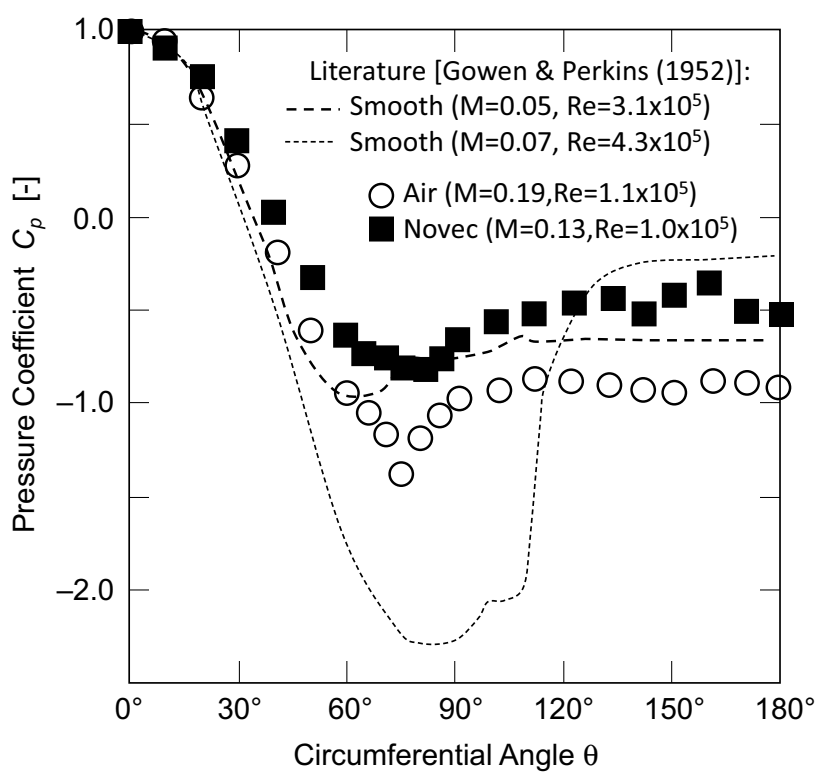

Fig. 5 Pressure coefficient $C_{p}$ against $\theta$ at low-speed flow and comparison with literature data for a smooth cylinder 


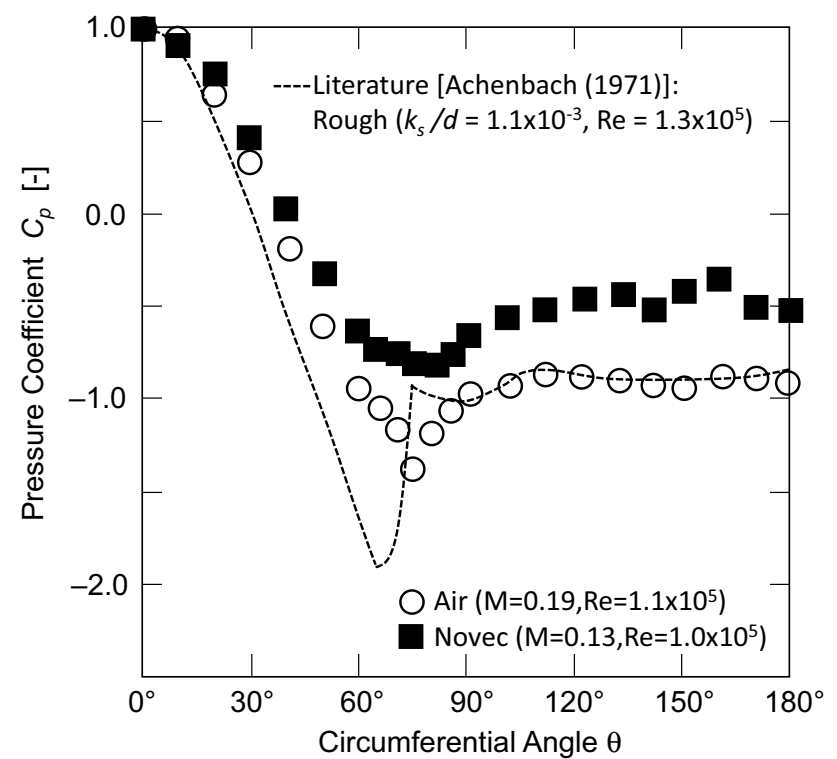

Fig. 6 Pressure coefficient $C_{p}$ against $\theta$ for low-speed flow and comparison with literature data from Achenbach (1971) obtained for a cylinder with similar relative roughness

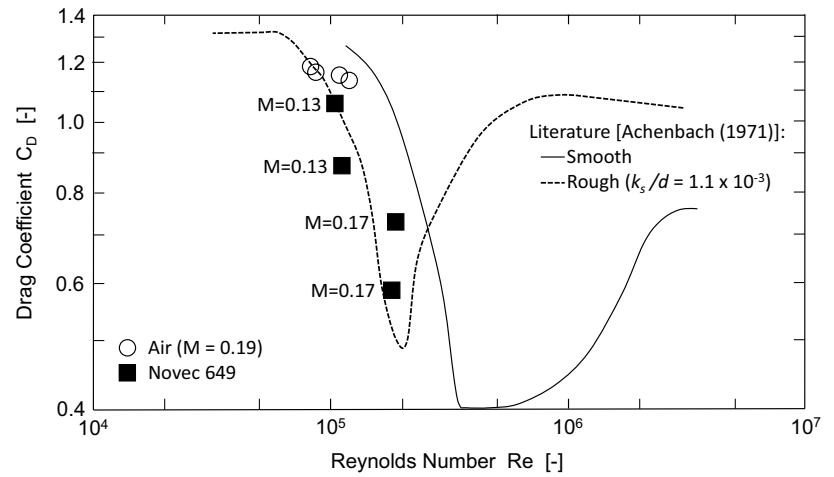

Fig. 7 Drag coefficient $C_{D}$ against Re for low-speed flow and comparison with literature results

typical literature results obtained for a smooth and a rough cylinder with a similar level of $k_{s} / d$. The results are shown in Fig. 7, and they strongly supported the observation that the actual cylinder surface was rather rough in terms of $k_{s} / d$ : All new data points were in excellent agreement with literature results published by Achenbach (1971) obtained at similar roughness and inflow turbulence levels. In contrast, literature (e.g., White (2006)) predicts a much higher critical Reynolds number for a smooth cylinder (the critical Reynolds number is defined here as the Reynolds number for which a significant drop of $C_{D}$ occurs due to transition and change in the wake flow). Since the present measurements were done in the transitional regime, the high sensitivity of the pressure distribution on minor changes in inflow conditions might explain the deviations between air and Novec data as shown in Fig. 6.

As expected, no systematic influence of the working fluid selection was observed in essentially incompressible flow. The data points corresponding to air and Novec ${ }^{\mathrm{TM}} 649$ measurements were consistent and followed virtually the literature correlation for a rough cylinder. This was investigated in more detail using the base pressure coefficient $C_{p B}=C_{p}\left(\theta=180^{\circ}\right)$, see Fig. 8. Although there was a significant data scattering, no systematic effect of the compressibility factor $Z$ on the base pressure behavior was observed. The results plotted in Fig. 8 demonstrated once again that the surface roughness of the present cylinder device was of some importance because the increase in the base pressure coefficient (which is directly connected to the decrease of the drag coefficient) was observed at a lower critical Reynolds number level than in case of smooth cylinders.

\subsection{High-speed observations}

Due to the comparable low speed of sound (see Table 1), it was possible to achieve Mach numbers of $M=0.6$ in the case of Novec ${ }^{\mathrm{TM}} 649$ in the present test facility, but due to compressor volume flow rate restrictions, it was not possible to achieve such a high Mach number level in air flows. Hence, the new data obtained for Novec ${ }^{\mathrm{TM}} 649$ can only be compared with compressible airflow results from other researchers.

Since the first measurements by Matt (1943), it is known that different flow phenomena interact at moderate Mach numbers of about $M=0.4$. Compressible flow phenomena become visible (which might be described by Mach number $M$ ), but in this moderate Mach number regime, there is still the incompressible flow phenomenon observable of transition and drop of drag coefficient at a critical Reynolds number. A qualitative discussion of these effects can be found

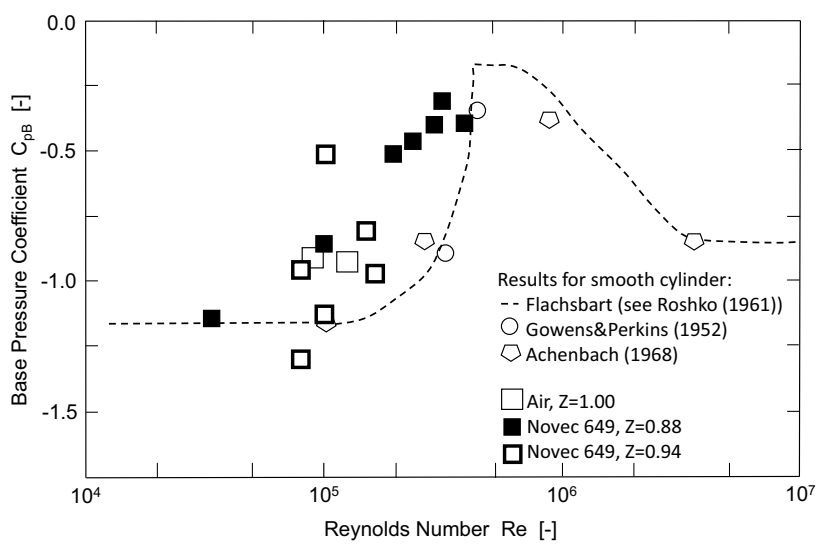

Fig. 8 Base pressure coefficient $C_{p B}$ against Re for low-speed flow and comparison with literature results 
in Naumann and Pfeifer (1962) and Ackerman et al. (2008). In Fig. 9, pressure coefficient data obtained for Novec ${ }^{\mathrm{TM}}$ 649 at two different compressibility factor levels are compared with available literature results for air (Ackerman et al. (2008)). It should be noticed that Ackerman et al. (2008) considered a much higher Reynolds number than the present study. Furthermore, Ackerman et al. (2008) used a hydraulically smooth cylinder with a blockage ratio of only half of the present study. These systematic differences between the experiments can explain the deviations between the new data and the literature data regarding base pressure. Interestingly, two Novec ${ }^{\mathrm{TM}} 649$ data sets agreed well with the literature data up to the separation position, whereas one Novec ${ }^{\mathrm{TM}}$ 649 data set $\left(Z=0.88, M=0.41, \operatorname{Re}=4.6 \cdot 10^{5}\right)$ exhibited a substantially weaker surface pressure minimum at about $\theta=90^{\circ}$. This might be an indication for a strong sensitivity to Reynolds number effects at this moderate Mach number level, which was noticed earlier by Naumann and Pfeifer (1962).

At a higher Mach number level of about $M=0.6$, several researchers published results for the pressure distribution of a cylinder in an air stream, and some of them are shown in Fig. 10 together with new data for Novec ${ }^{\mathrm{TM}}$ 649. The overall agreement between the new data was rather good. Furthermore, the compressibility factor $Z$ seemed to be of minor importance (only at the minimum pressure region, a slight effect was observed; as discussed in more detail in the following Sect. 3.3 in connection with the critical pressure coefficient $C_{p, \mathrm{cr}}$ ).

In Fig. 11, form drag coefficients $C_{D}$ based on surface pressure measurements in air and Novec ${ }^{\mathrm{TM}} 649$ are

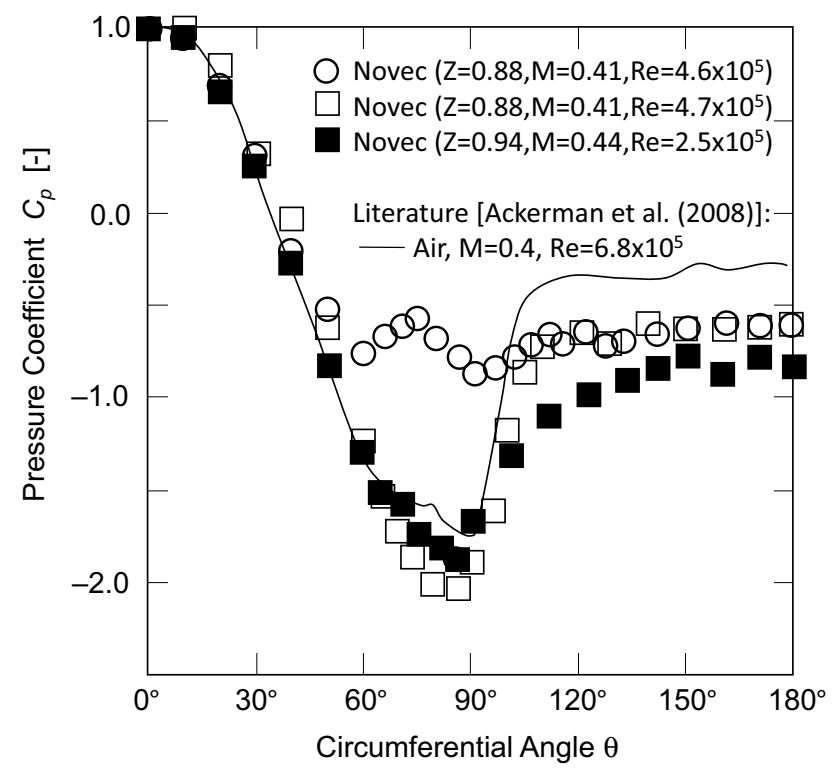

Fig. 9 Pressure coefficient $C_{p}$ against $\theta$ at $M=0.4$ and comparison with literature data obtained for a smooth cylinder

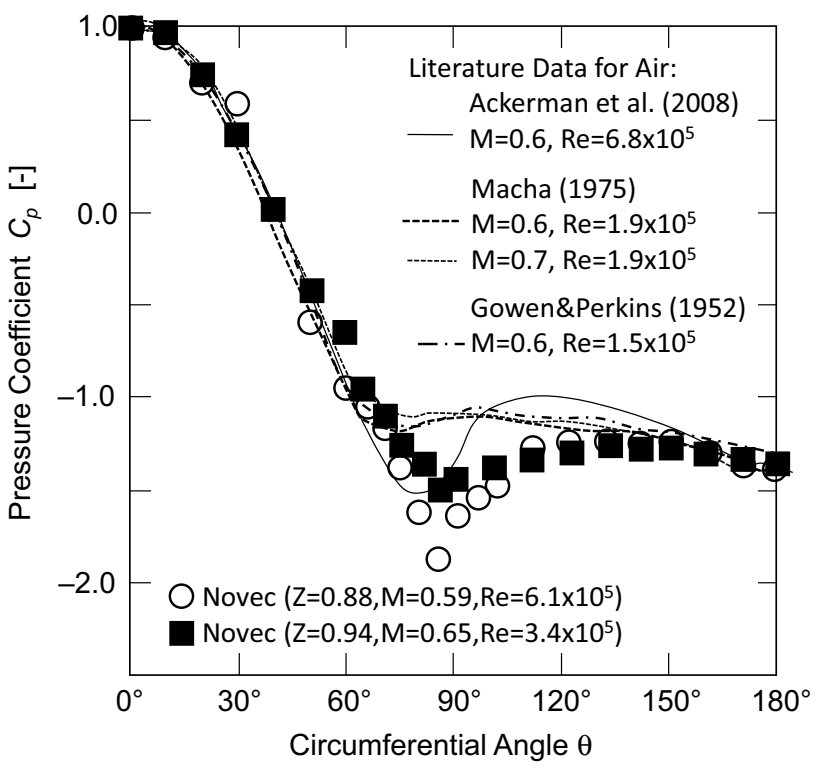

Fig. 10 Pressure coefficient $C_{p}$ against $\theta$ at $M=0.6$ and comparison with literature data obtained for a smooth cylinder

compared for a range of Reynolds and Mach numbers. In the case of low Mach numbers $(M<0.2)$, it was found in the previous Sect. 3.1 that the compressibility factor $Z$ was not relevant and that the drag coefficient followed the literature correlation proposed for a cylinder with similar roughness. The behavior of the drag coefficient $C_{D}$ as a function of Reynolds number Re demonstrated that the present organic vapor flow investigation at $M=0.4$ was performed within the transitional regime. This explained the strong sensitivity against the Reynolds number for this Mach number (see Fig. 9). Remarkably, the organic vapor results and the literature results for air followed a line similar to the well-known drag-crisis line observed for the cylinder. The shift of the

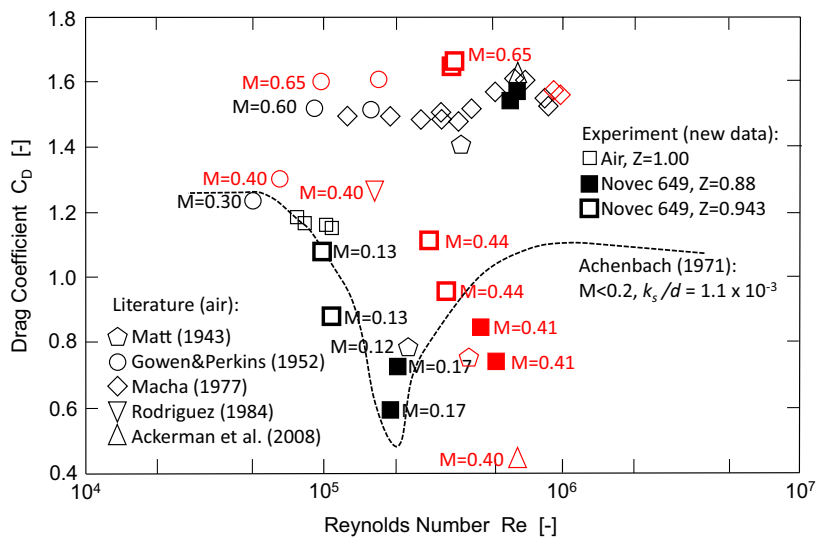

Fig. 11 Drag coefficient $C_{D}$ against Re for several Mach numbers $M$ and comparison with available literature results obtained for air (colored symbols indicate different Mach number clusters) 
critical Reynolds number to higher values in the case of $M=0.4$ was already observed in the case of airflow past a sphere (see Schlichting and Gersten (1997). As discussed by Naumann and Pfeifer (1962), at $M=0.6$, compressibility effects become dominant, and there was no significant drag crisis visible in the corresponding data shown in Fig. 11. The new Novec ${ }^{\mathrm{TM}} 649$ data agreed well with literature results obtained for air, and even the effect of an increase in the form drag coefficient $C_{D}$ at $\operatorname{Re} \approx 6 \times 10^{5}$ was observed for both fluids. This Mach number level is investigated in more detail in Fig. 12 and Fig. 13. In Fig. 12, the original data obtained for Novec ${ }^{\mathrm{TM}} 649$ are compared with uncorrected literature results. The overall agreement was good at $M=0.6$ (only the single data point of Matt (1943) was somewhat lower than the data of the other researchers). As discussed in Sect. 2.5, blockage corrections are of some relevance in this flow regime, and the uncorrected data of Fig. 12 were corrected regarding blockage effects. This led to a slightly better agreement between the new organic vapor flow data and the literature air flow data as demonstrated in Fig. 13. The observation of an increase in the drag coefficient $C_{D}$ between a Reynolds number range of about $5 \times 10^{5}<\operatorname{Re}<8 \times 10^{5}$ was supported by the new measurements.

In Fig. 14, the drag coefficient $C_{D}$ obtained at different Reynolds numbers Re is plotted against Mach number $M$. The general trend (i.e., a slight increase in drag coefficient with increasing Mach number between $M=0$ up to $M=0.5$ and local maximum at $M=0.6$ ) can be identified in Fig. 14 although the data scattering was significant due to the different Reynolds numbers. The comparison with literature data indicated that no extraordinary effect due to non-ideal gas dynamics was present up to $M=0.65$ : the new data points were well within the literature results obtained for an ideal gas (air).

As mentioned in the introduction, knowledge about the base pressure coefficient is of importance for turbomachinery

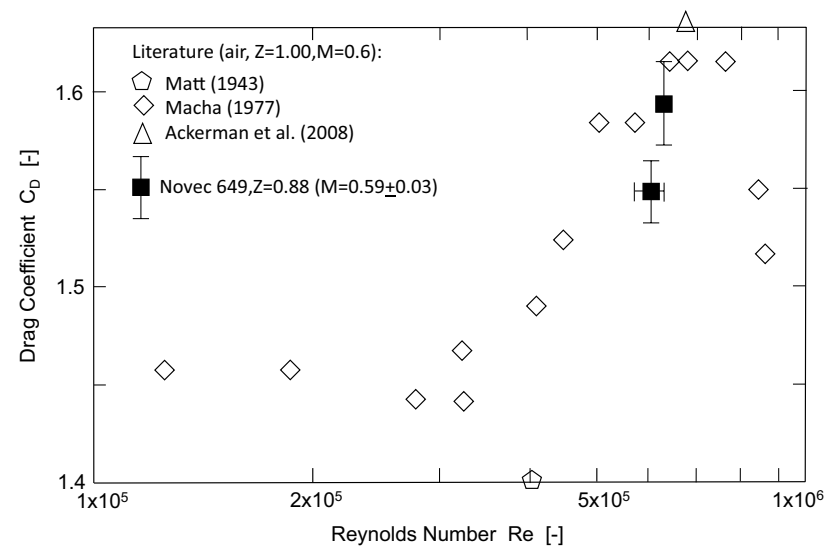

Fig. 12 Drag coefficient $C_{D}$ against $\operatorname{Re}$ at $M=0.6$ and comparison with available literature results obtained for air (uncorrected data)

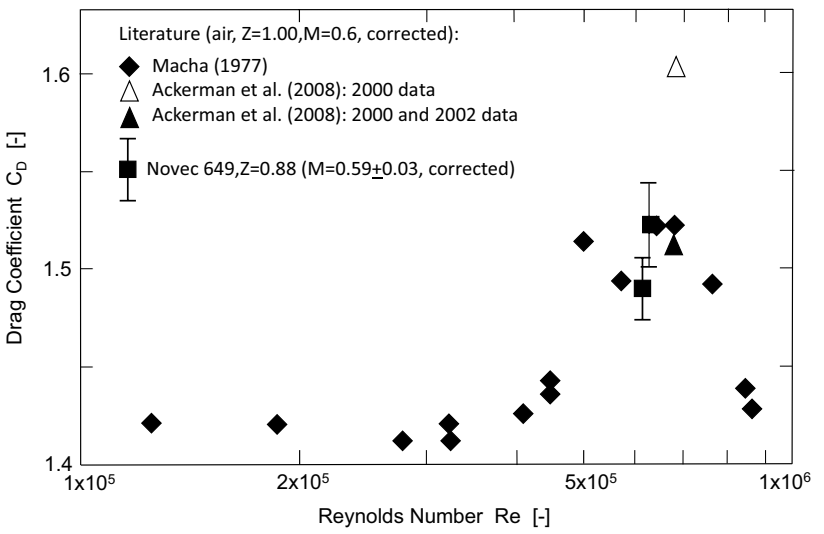

Fig. 13 Blockage-corrected drag coefficient $C_{D}$ against Re at $M=0.6$ and comparison with available literature results obtained for air (corrected data)

applications. Figure 15 shows the base pressure coefficients $C_{p B}$ obtained for Novec ${ }^{\mathrm{TM}} 649$ against Reynolds number Re for three different Mach numbers $M$. In the case for which literature data (obtained for air) were available, these data are plotted in Fig. 15, too. For a moderate Mach number $M=0.4$, a nearly linear increase in $C_{p B}$ with Re was observed. The result of Ackerman et al. (2008) can be interpreted as an extrapolation of the new data set in the case of $M=0.4$. At $M=0.5$, the available data did not allow a clear interpretation: It seemed to be the case that the base pressure coefficient $C_{D B}$ was nearly constant, but a single point at about $\operatorname{Re}=5 \times 10^{5}$ was higher than the other points obtained for $M=0.5$. Based on these few data points, it is not possible to decide if this could be the result of a fundamental Reynolds number effect. At a higher Mach number level, $M=0.6$,

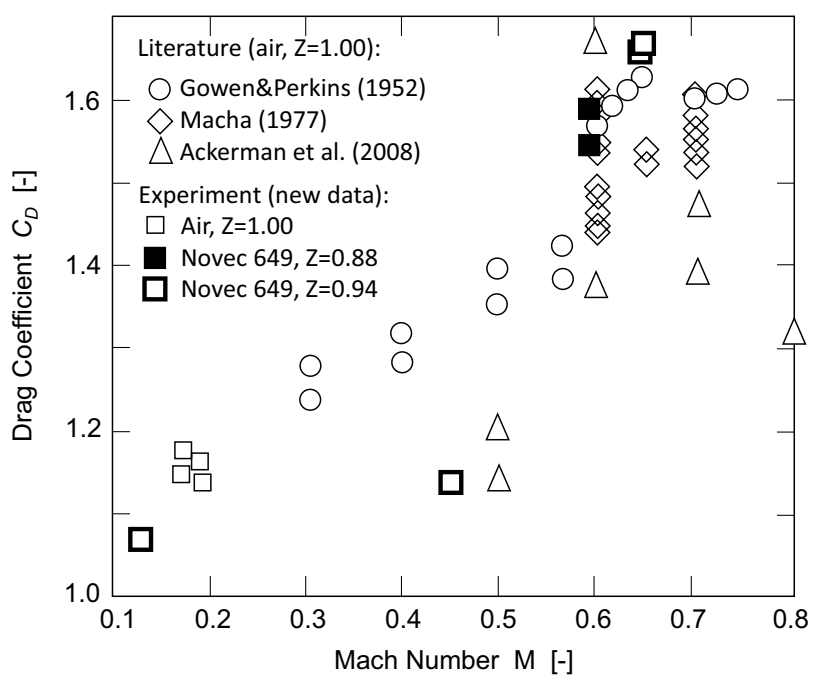

Fig. 14 Drag coefficient $C_{D}$ against $M$ (different Re) and comparison with available literature results obtained for air (uncorrected data) 


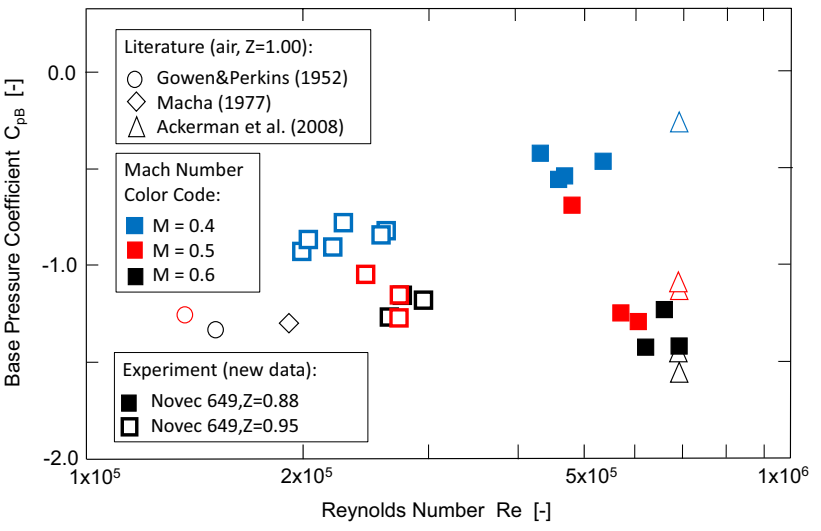

Fig. 15 Base pressure coefficient $C_{p B}$ against Re at different Mach number levels and comparison with literature results

the data indicated a weak influence of the Reynolds number (there was a tendency that the base pressure coefficient decreased slightly with the Reynolds number). To investigate the base pressure behavior in more detail, all available results for $C_{p B}$ are plotted against Mach number $M$ for sufficient high Reynolds numbers Re in Fig. 16. In Fig. 16, only results for $\operatorname{Re}>1.3 \times 10^{5}$ are shown because at least in the case of low Mach numbers, the subcritical flow would lead to completely different results for lower Reynolds numbers. The data shown in Fig. 16 indicated that for sufficiently high Reynolds numbers, two base pressure coefficient regimes can be assumed: for low and moderate Mach numbers $(M<0.5)$, the base pressure coefficients were relatively high, but at $M=0.5$, a sharp drop of $C_{p B}$ was observed. This observation agrees well with the other findings that at $M=0.6 \mathrm{com}$ pressibility effects become dominant. The transition between these two regimes occurred at about $M \geq 0.5$. Interestingly, no systematic influence of the compressibility factor $Z$ or the isentropic exponent $\kappa$ on the flow transition point was observed during the present experiments.

\subsection{Discussion}

In aerodynamics (see, for instance, Anderson (2010)), the so-called critical pressure coefficient $C_{p, \mathrm{cr}}$ as a function of the free-stream Mach number $M$ is of practical interest. The critical pressure coefficient $C_{p, \text { cr }}$ is defined as the value for which Mach 1 would be achieved at the surface of the body subjected to a stream of a compressible fluid with freestream Mach number $M$ and dynamic pressure $q$. Using definition (1), the critical pressure coefficient

$C_{p}=\frac{p_{w}-p}{q}=\frac{p}{q}\left(\frac{p_{w}}{p}-1\right)=\frac{p}{q}\left(\frac{p_{w}}{p_{0}} \frac{p_{0}}{p}-1\right)$

can be obtained directly as function of the isentropic relations $f_{i s}(M)=p_{0} / p$ and $f_{i s}(M=1)=p_{0} / p_{w}$. In the case of an ideal gas, an analytical expression

$C_{p, \mathrm{cr}}=\frac{2}{\kappa M^{2}}\left(\left(\frac{2+(\kappa-1) M^{2}}{\kappa+1}\right)^{\kappa /(\kappa-1)}-1\right)$

results, whereas in the case of a non-ideal gas the isentropic relations have to be evaluated based on a suitable equation of state (see, for instance, Passmann et al. (2017)). In Fig. 17, the critical pressure coefficient $C_{p, \text { cr }}$ against free-stream Mach number $M$ is plotted for air (ideal gas with $\kappa=1.40$ ) and Novec ${ }^{\mathrm{TM}} 649$ (real gas) at temperature and pressure conditions as representative for the present experiments. In
Fig. 16 Base pressure coefficient $C_{p B}$ against Mach number $M$ at higher Reynolds number level and comparison with literature results

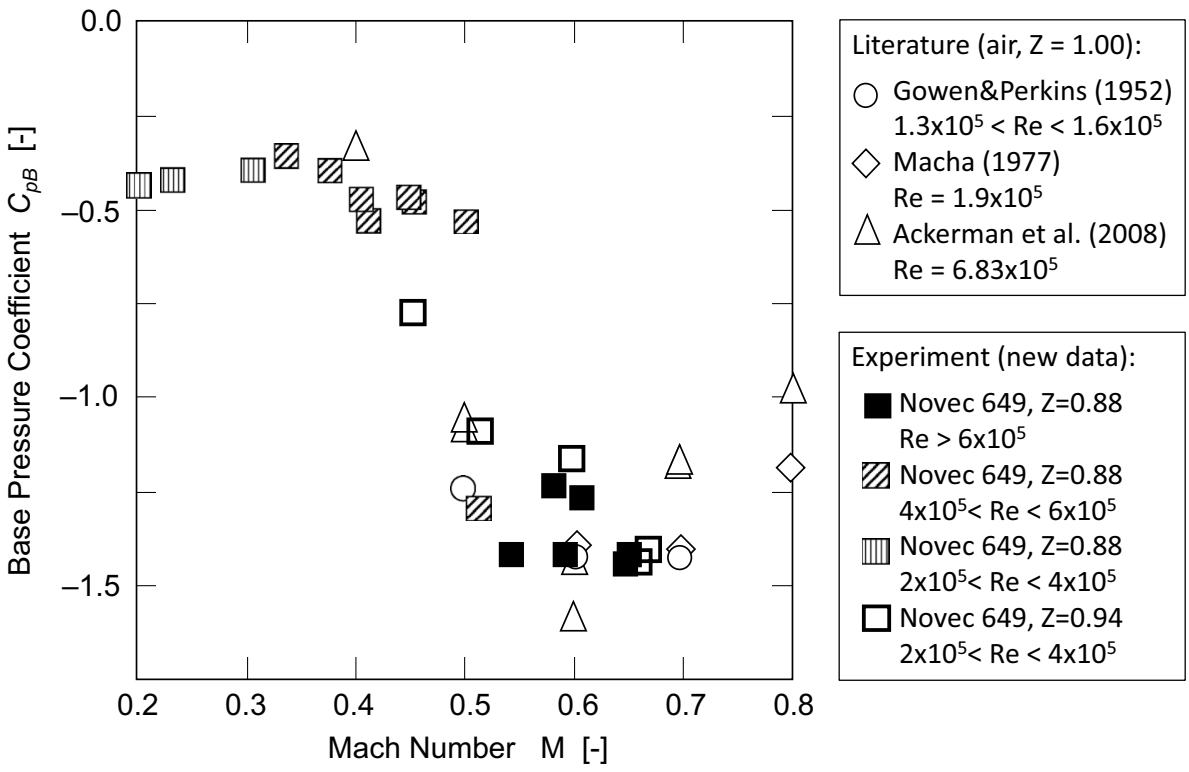




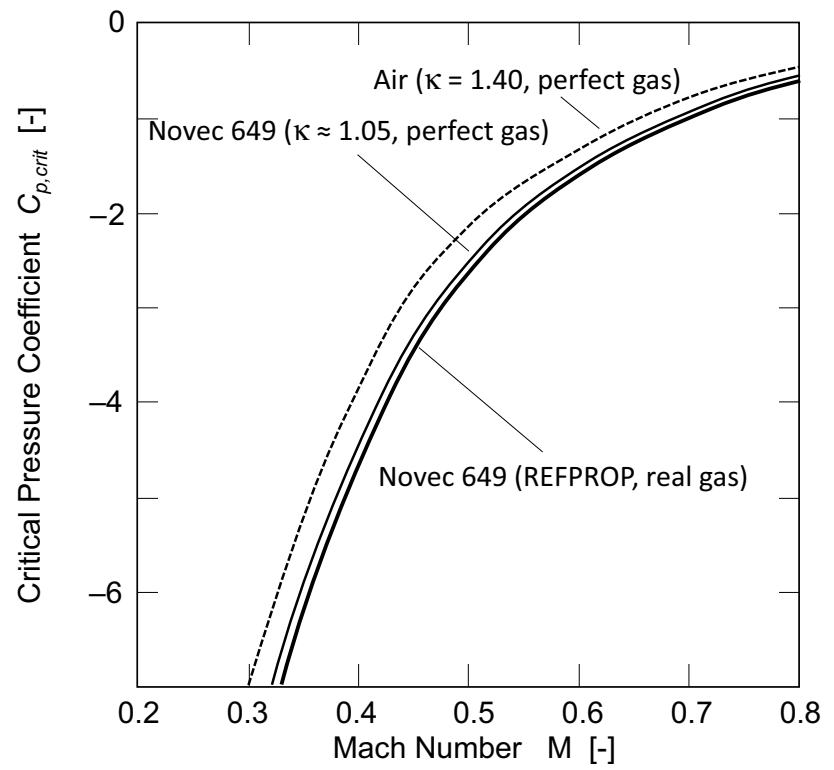

Fig. 17 Critical pressure coefficient $C_{p, c r i t}$ against Mach number $M$ for air and Novec ${ }^{\mathrm{TM}} 649$ at conditions listed in Table 1

the case of Novec ${ }^{\mathrm{TM}} 649$, the critical pressure coefficient was calculated using two different methods, namely by a direct calculation using REFPROP and assuming an isentropic expansion from a defined reservoir condition, and also by using the simple ideal-gas model, Eq. (12), with an appropriate isentropic exponent of about $\kappa \approx 1.05$, see Table 1. The deviations between the two calculation methods for Novec ${ }^{\mathrm{TM}} 649$ were not substantial. The behavior of the critical pressure coefficient was also rather similar for air and organic vapor. Based on the present measurements, a conclusion could be that real gas effects might be of only minor importance in high subsonic flow and the general flow mechanisms remain mainly the same (but it should be noted that the range of investigated reservoir conditions was limited). However, there was a systematic difference between the critical pressure coefficients for air and Novec ${ }^{\mathrm{TM}}$ 649, Fig. 17, and this difference influenced the local pressure distribution close to the critical position. This is shown in more detail through Fig. 18 where pressure coefficient $C_{p}$ against circumferential angle $\theta$ is plotted in the vicinity of the pressure minimum. In addition to the pressure coefficient $C_{p}$, the values of the critical pressure coefficient $C_{p, c r}$ are indicated as dotted lines in Fig. 18. The critical pressure coefficient $C_{p, c r}$ for Novec ${ }^{\mathrm{TM}} 649$ was noticeably lower than for air, and this difference was of the same order as the difference of the minima in the $C_{p}$ distributions for air and Novec ${ }^{\mathrm{TM}} 649$. In both cases, the observed $C_{p}$ minimum was close to the value for the critical pressure coefficient $C_{p, c r}$ (but the actual $C_{p}$ minima were systematically lower than the $C_{p, c r}$ values). This detailed investigation led to the conclusion, that nonideal gas effects locally affect the flow past a cylinder in high

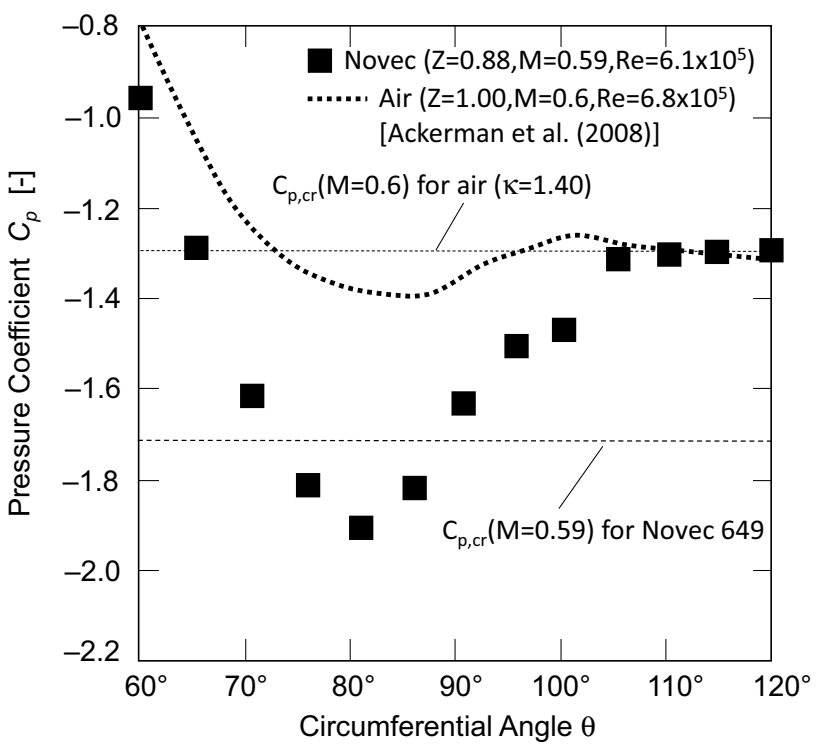

Fig. 18 Pressure coefficient $C_{p}$ against $\theta$ at $M=0.6$ for air (literature data) and Novec ${ }^{\mathrm{TM}} 649$ in the flow separation region and corresponding critical pressure coefficient values (broken horizontal lines)

subsonic flow, but this does not contribute significantly to the overall form drag coefficient $C_{D}$ because the contribution of $C_{p}$ at $\theta \approx 90^{\circ}$ is nearly negligible in the evaluation of the integral expression (2).

Following Ackerman et al. (2008), it is instructive to compare the form drag coefficient $C_{D}$ (see Eq. (2)) with the base pressure drag coefficient $C_{D B}$ (see Eq. (3)). This comparison is shown graphically in Fig. 19 for some new data obtained for Novec ${ }^{\mathrm{TM}} 649$ and literature data

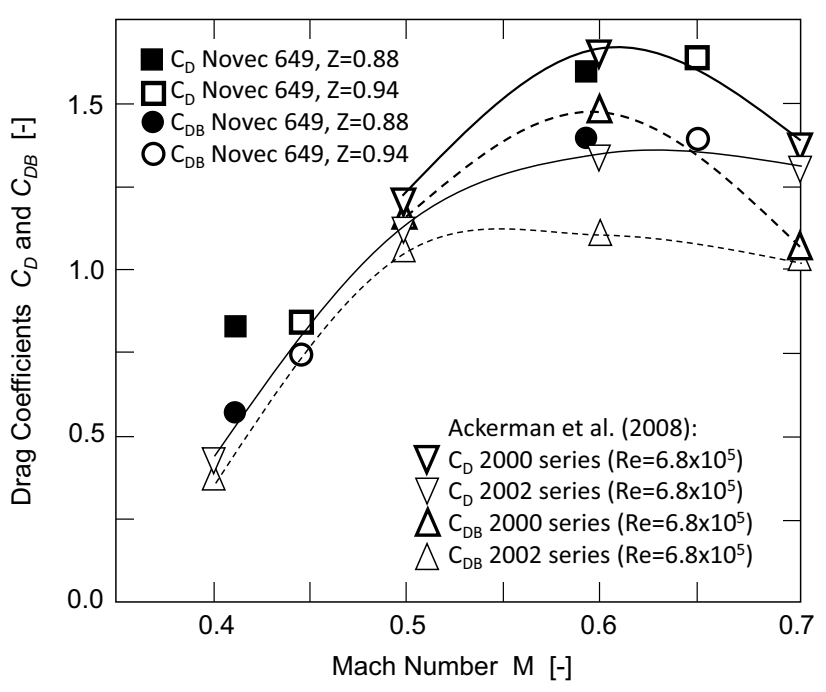

Fig. 19 Drag coefficient $C_{D}$ and base pressure drag coefficient $C_{D B}$ against $M$ comparison with literature results reported for air (Ackermann (2008)) 
obtained for air. In incompressible flow past a cylinder, it is known that the base pressure drag contributes substantially to the total form drag. In the case of compressible flow, the time-averaged pressure distribution is also affected by compressibility effects. Before considering the differences between the data sets plotted in Fig. 19, an explanation of the changes in $C_{D}$ over the Mach number $M$ will illustrate the reason for the differences. From Fig. 19 it can be seen that both $C_{D}$ and $C_{D B}$ start low and then raise to a maximum at about $M=0.6$, before falling off at $M=0.7$. These variations over the Mach number range are compatible with the compressible flow regimes described by Zdravkovich (2003), namely, shock-less, intermittent shock wave, and permanent shock wave (a fourth flow regime referred to as wake shock wave would occur at higher Mach number). At $M=0.4$, the flow is in the shock-less regime, and it is strongly dependent on Reynolds number; it is this effect that determines $C_{D}$. The new Novec ${ }^{\text {TM }} 649$ data point at $M=0.41$ was obtained at a much lower Reynolds number $\left(\operatorname{Re}=4.6 \times 10^{5}\right)$ than the air value by Ackerman et al. (2008). In this case, given the Reynolds number used in the test, the flow falls into the so-called Transitional Boundary Layer 3 (TrBL3) subsonic regime (Zdravkovich (2003)). Just above $M=0.4$ the (air) flow becomes critical, i.e., local regions of flow around the cylinder become supersonic and the flow enters the intermittent shock wave regime. The oscillating flow field present in this regime, which results in the flow being supersonic only on one side of the cylinder at a time, leads to increased pressure fluctuations compared to the previous Mach number. Following Ackerman et al. (2008), the increase in $C_{D}$ leading up to $M=0.6$ is a result of the commencement of vortex shedding. Beyond around $M=0.65$, the flow enters the permanent shock wave regime. The permanent shock wave regime causes the movement of the location of formation and shedding of the vortices downstream of the cylinder surface. This lengthens the formation region, increases the pressure recovery, and gives a slightly earlier separation. This in turn leads to the reduction in $C_{D}$ at $M=0.7$. The new results for the organic vapor flow were in reasonable agreement with literature data obtained for air; the deviations of the data points in Fig. 19 can be already explained by Reynolds number effects or experimental uncertainty range. The deviations between the organic vapor flow and literature data were of the same order as the scattering of the literature data (Ackerman et al. (2008) performed two series of essentially identical experiments in 2000 and 2002). This led to the conclusion that non-ideal gas effects were of minor importance for the overall flow behavior at the considered Mach number range.

\section{Conclusion and outlook}

A circular cylinder was tested in the cross-flow of an organic vapor (Novec ${ }^{\mathrm{TM}}$ 649) and air over the subsonic and high subsonic speed range in a continuously running closed wind tunnel test facility. Time-averaged pressure measurements gave information on surface pressure distributions, and the corresponding drag and base drag coefficients were obtained. Due to charging of the test facility, different values for the compressibility factor could be achieved for the organic vapor flow, which together with the results for air enabled an assessment of the impact of non-ideal gas dynamics on the form drag of a cylinder in high subsonic flow.

The following conclusions can be drawn: The cross-flow about a circular cylinder changes significantly at higher subsonic velocities and into the transonic range. It was found that non-ideal gas effects did not strongly affect the overall behavior regarding time-averaged cylinder surface pressure distribution and form drag. The variation of drag over the Mach number range was compatible with the common understanding of ideal gas compressible flow regimes, namely, shock-less, intermittent shock wave, and permanent shock wave. At Mach 0.4, the flow is in the shock-less regime, and it is strongly Reynolds number dependent. An increase in drag was observed at Mach 0.6 , which was attributed to the commencement of vortex shedding, and real-gas thermodynamics affected only locally the flow due to the shift of the critical pressure coefficient.

To investigate the local non-ideal flow phenomena in more detail, time-resolved pressure distributions and high-speed schlieren optical methods might be of great value. Such an investigation is the subject of future research. Furthermore, it is planned to extend the investigations to the transonic and supersonic flow regime by designing a new transonic test section including a supersonic nozzle.

Author contributions All authors contributed to the study conception and design. SadW and FR planned details of the research project, and FR was the project leader and conducted the experimental study. RW and LH supported the design of the test section and the operation of the test facility during the measurements. SadW and FR performed the data reduction and data interpretation including detailed uncertainty analysis. The first draft of the manuscript was written by SadW and all authors commented on previous versions of the manuscript. All authors read and approved the final manuscript.

Funding Open Access funding enabled and organized by Projekt DEAL. Development and erection of the test facility which was used in the present study were financially supported by the German BMWi program "Ingenieurnachwuchs" under the project name "LES for ORC" between 2013 and 2017.

\section{Compliance with ethical standards}

Conflicts of interest The authors declare that they have no conflict of interest. 
Availability of data and material Experimental data and further information can be sent on request by the corresponding author.

Open Access This article is licensed under a Creative Commons Attribution 4.0 International License, which permits use, sharing, adaptation, distribution and reproduction in any medium or format, as long as you give appropriate credit to the original author(s) and the source, provide a link to the Creative Commons licence, and indicate if changes were made. The images or other third party material in this article are included in the article's Creative Commons licence, unless indicated otherwise in a credit line to the material. If material is not included in the article's Creative Commons licence and your intended use is not permitted by statutory regulation or exceeds the permitted use, you will need to obtain permission directly from the copyright holder. To view a copy of this licence, visit http://creativecommons.org/licenses/by/4.0/.

\section{References}

Achenbach E (1971) Influence of surface roughness on the cross-flow around a circular cylinder. J Fluid Mech 46(2):321-335

Ackerman, J R, Gostelow, J P, Rona, A, Carscallen, W E (2008) Base Pressure Measurements on a Circular Cylinder in Subsonic Cross Flow, AIAA Conference paper, AIAA2008-4305

Allen HJ, Vincenti WG (1944) Wall interference in a two-dimensionalflow wind tunnel, with consideration of the effect of compressibility. Adv Comm Aero, Wash, Rep, Nat, p 782

Anders, J B (1993) Heavy Gas Wind Tunnel Research at Langley Research Center, ASME Paper 93-FE-5.

Anderson JD (2010) Introduction to Flight. McGraw-Hill, New York

Cogswell, F J, Gerlach, D W, Wagner, T C, Mulugeta J. (2011)) Design of an Organic Rankine Cycle for Waste Heat Recovery From a Portable Diesel Generator, Proceedings ASME 2011 International Mechanical Engineering Congress and Exposition, DOI: https:// doi.org/10.1115/IMECE2011-65489

Cozzi F, Spinelli A, Carmine M, Cheli R, Zocca M, Guardone A (2015) Evidence of complex flow structures in a converging- diverging nozzle caused by a recessed step at the nozzle throat, 45th AIAA fluid dynamics conference, Dallas, 22-26 June 2015

Dejc ME, Trojanovskij BM (1973) Untersuchung und Berechnung axialer Turbinenstufen. VEB Verlag Technik, Berlin

Gowen FE, Perkins EW (1952) Drag of circular cylinders for a wide range of Reynolds numbers and Mach numbers, NACA Report RM A52C20, Moffett Field, CA

Hasselmann K, aus der Wiesche, S, Kenig, E Y, (2019) Optimization of Piecewise Conical Nozzles: Theory and Application. ASME J Fluids Eng 141(12):121202

Kouremenos DA, Antonopoulos KA (1986) Isentropic Exponents of Real gases and Application for the Air at Temperatures From 150 K to $450 \mathrm{~K}$. Acta Mech 65:81-99

Macha JM (1977) Drag of Circular Cylinders at Transonic Mach Numbers. J Aircraft 14(6):605-607

MacMartin, I P and Norbury, J F (1974) The Aerodynamics of a Turbine Cascade with Supersonic Discharge and Trailing Edge Blowing, ASME Paper 74-GT-120

Matt, H (1943) Measurement of Drag of Round Rods of Various Diameters at High Subsonic Airspeed, Deutsche Luftfahrtforschung, Forschungsberichte (Report) No. 1825, 1943 (in German)
McLinden et al (2015) (2015) Thermodynamic Properties of 1,1,1,2,2,4,5,5,5-Nonafluoro-4(trifluoromethyl)-3-pentanone: Vapor Pressure, (p, $\rho, T)$ Behavior, and Speed of Sound Measurements, and an Equation of State. J Chem Eng Data 60:3646-3659

Murthy VS, Rose WC (1978) Detailed Measurements on a Circular Cylinder in Cross Flow. AIAA Journal 16:549-550

Naumann A, Pfeifer H (1962) Über die Grenzschichtströmung am Zylinder bei hohen Geschwindigkeiten. In: von Karman T (ed) Advances in Aeronautical Sciences, vol 3. Pergamon Press, New York, pp 185-206

Passmann, M, Reinker, F, Hasselmann, K, aus der Wiesche, S, Joos, F (2016) Development and Design of a Two-Stage Contraction Zone and Test Section of an Organic Rankine Cycle Wind Tunnel, Proceeding ASME Turbomachinery Technical Conference and Exposition, June 13-17, 2016, Seoul, South Korea, Paper No: GT2016-56580, V003T25A006; 11 pages

Passmann M, Aus der Wiesche S, Joos F (2017) A one-dimensional analytical calculation method for obtaining normal shock losses in supersonic real gas flows. J Phys Conf Ser 821(1):012004

Reinker, F, Kenig, E Y, aus der Wiesche, S (2018) CLOWT: A Multifunctional Test Facility for the Investigation of Organic Vapor Flows, Proceedings ASME 2018 5th Joint US-European Fluids Engineering Division Summer Meeting, Montreal, Canada (V002T14A004).

Reinker, F, Kenig, E Y, aus der Wiesche, S (2019) Closed Loop Organic Vapor Wind Tunnel CLOWT: Commissioning and Operational Experience, Proceeding ORC 2019 Athens, Greece, paper-ID 47

Reinker, F, Wagner, R, Passmann, M, Hake, L, aus der Wiesche, S (2020) Performance of a Rotatable Cylinder Pitot Probe in High Subsonic Non-Ideal Gas Flows, Proceedings 3rd Symposium NICFD, TU Delft

Reinker, F, aus der Wiesche, S (2020) Application of Hot-Wire Anemometry in the High Subsonic Organic Vapor Flow Regime, Proceedings 3rd Symposium NICFD, TU Delft

Rodriguez O (1984) The Circular Cylinder in Subsonic and Transonic Flow. AIAA Journal 22(12):1713-1718

Roshko A (1961) Experiments on the flow past a circular cylinder at very high Reynolds number. J Fluids Mechanics 10(3):345-356

Rotta JC (1972) Turbulente Strömungen. Teubner, Stuttgart

Schlichting H, Gersten K (1997) Grenzschicht-Theorie, 9th edn. Springer, Berlin

Spinelli A, Cammi G, Gallarini S, Zocca M, Cozzi F, Gaetani P, Dossena V, Guardone A (2018) Experimental evidence of non-ideal compressible effects in expanding flow of a high molecular complexity vapor. Exp Fluids 59:126

Stratford BS (1959) The prediction of separation of the turbulent boundary layer. J Fluid Mech 5:1-16

White FM (2006) Viscous Fluid Flow, 3rd edn. McGraw-Hill, New York

Wyler JS (1975) Probe Blockage Effects in Free Jets and Closed Tunnels. ASME J Eng Power 97(4):509-514

Zdravkovich MM (2003) Flow around Circular Cylinders 1:566-571

Publisher's Note Springer Nature remains neutral with regard to jurisdictional claims in published maps and institutional affiliations. 